\title{
ON SOME ASYMPTOTIC PROPERTIES CONCERNING HOMOGENEOUS DIFFERENTIAL PROCESSES
}

\author{
TUNEKITI SIRAO
}

1. Introduction. About the behaviour of brownian motion at time point $\infty$ there are many results by P. Lévy and A. Khintchine etc. The method of W. Feller ${ }^{11}$ is applicable to a similar discussion about a homogeneous differentia! process. In this paper we shall study, applying his method, the properties of a homogeneous differential process.

Let $\{X(t, \omega) ; 0 \leqq t<\infty, \omega \in \Omega\}^{2)}$ be a homcgenecus differential prccess such that $E(X(t))=m t$ and $V\left(X^{\prime}(t)\right)=\sigma^{2} t$. $^{3)}$ After P. Lévy we shall define the concept of upper class and lower class with respect to a homogeneous differential process as follows: if the set of $t$ such that

$$
X(t, \omega)>\sigma \sqrt{t} \phi(t)
$$

is bounded (unbounded) for almost all $\omega$, then we say that $\psi(t)$ belongs to the upper (lower) class with respect to $\{X(t) ; 0 \leqq t<\infty\}$. Then we may prove the following three theorems. In these theorems, the distribution function of $X(t)$ is denoted by $V_{t}(x)$.

Theorem 1. Let $\{X(t, \omega) ; 0 \leqq t<\infty, \omega \in \Omega\}$ be a right continuous ${ }^{4}$ homogeneous differential process satisfying the following conditions:

$$
\int_{|x-m|<z}|x-m|^{3} d V_{1}(x)=\mathrm{O}\left(z(\log \log z)^{-1 / 2}\right) \quad \text { as } \quad z \rightarrow \infty .
$$

For any $\varepsilon>0$,

$$
\int_{|x-m|<-z}(x-m)^{2} d V_{1}(x)=\mathrm{O}\left((\log \log z)^{-(2+\varepsilon)}\right)
$$

or

$$
\int_{\{x-m<z}(x-m)^{2} d V_{1}(x)=o\left((\log \log z)^{-2}\right) \quad \text { as } \quad z \rightarrow \infty .
$$

Received April 30, 1953.

1) W. Feller: "The law of the iterated logarithm for identically distributed random variables." Ann. of Math. vol. 47 (1946).

2) $\omega$ is the probability parameter.

3) The symbols $E$ and $V$ denote the expectation and the variance respectively.

1) This is not an essential restriction. 
There exist two bositive iumbers $\alpha$ and $N$ such that, for $0 \leqq t \leqq \alpha^{5)}$ and $0<N \leqq b-a$,

$$
\int_{a \leqq i x-m t \mid<b} d V_{t}(x)=\mathrm{O}\left(t \int_{a \leqq|x-m|<b} d V_{1}(x)\right) \text { as } \quad a \rightarrow \infty,
$$

uniformly in $t$.

Then a monotone non-decreasing right continuous funstion $\phi(t)$ belongs to the upper (lower) class if, and only if,

$$
\int^{\infty} \frac{1}{t} \phi(t) e^{-\frac{1}{2} \phi^{2}(t)} d t \in\left(S(D) .^{6)}\right.
$$

Example 1. For a Poisson process, the conditions (1), (2) and (3) are well satisfied.

Example 2. For a process of Pearson type, that is, a differential process $\{X(t, \omega) ; 0 \leqq t<\infty\}$ such that

$$
P_{r}\{X(t, \omega) \leqq x\}= \begin{cases}\int_{0}^{x} \frac{e^{-y} y^{t-1}}{\Gamma(t)} d y & \text { if } \quad x \geqq 0 \\ 0 & \text { otherwise, }\end{cases}
$$

the conditions (1), (2) and (3) are well satisfied.

Theorem 2. Let $\{X(t, \omega) ; 0 \leqq t<\infty, \omega \in \Omega\}$ be a right continuous homogeneous differential process with symmetric distribution function $V_{t}(x)$. Then, in Theorem 1, we may remove the assumption (1) and palliate (2) as follows:

$$
\int_{|x-m|>z}(x-m)^{2} a V_{1}(x)=\mathrm{O}\left((\log \log z)^{-1}\right)
$$

Example 3. For a Gaussian process, the conditions (2)" and (3) are well satisfied.

Theorem 3. Let $\{X(t, \omega) ; 0 \leqq t<\infty, \omega \in \Omega\}$ be a right continuous homogeneous differential process. If $E\left((X(t)-m t)^{4}\right)$ is finite, the criterion (4) is valid.

2. Proofs. Without loss of generality we may assume that $m=0$ and $\sigma=1$.

Lemma 1. Let $\phi(t)$ be a monotone non-decreasing right continuous function. If $\phi(t)$ does not belong to the upper class, then there exists a monotone increasing sequence $\left\{t_{k}\right\}$ such that $\left\{\phi_{k}=\phi\left(t_{k}\right)\right\}$ does not belong to the upper class with respect to $\left\{X_{k} ; X_{k}=X\left(t_{k}\right)-X\left(t_{k-1}\right)\right\}$.

Proof. Let $\phi(t)$ be a function which does not belong to the upper class. Then there exists a set $\Omega^{*} \equiv \Omega$ with positive probability such that, for any $T>0$,

5) We may assume $\alpha \leqq 1$ without losing generality.

6) $E(\mathfrak{D}(\mathfrak{D})$ denotes the convergence (divergence) of the integrals. 
there exists $t(\omega)>T$ such as

$$
X(t, \omega)>\sqrt{t} \phi(t) \text { when } \omega \in \Omega^{*} .
$$

Since $X(t, \omega)$ is right continuous in $t$, we have

$$
X(r, \omega)>\sqrt{r} \phi(r) \text { when } \omega \in \Omega^{*},
$$

with a rational number $\gamma(\omega)(>T)$. Let us put

$$
\operatorname{Pr}\left(\Omega^{*}\right)=c>0 \text {. }
$$

Let $\left\{r_{i}\right\}$ be the set of all rational numbers. We shall define $\Omega_{n}^{(1)}$ as follows:

$$
\Omega_{n}^{(1)}=\left\{\omega \in \Omega^{*} ;{ }^{\exists} r_{i} \leqq n, X\left(r_{i}, \omega\right)>\sqrt{r_{i}} \phi\left(r_{i}\right)\right\} \quad(n=1,2, \ldots),
$$

where " ${ }^{\exists} r_{i} \leqq n$ " means that there exists at least one $r_{i}$ which does not exceed $n$. Then we have, by (6) (with exception of the set of zero measure),

$$
\bigcup_{n} \Omega_{n}^{(1)}=\Omega^{*}, \quad \Omega_{1}^{(1)} \leqq \Omega_{2}^{(1)} \subseteq \ldots \leqq \Omega_{n}^{(1)} \cong \ldots .
$$

Hence, for any $\varepsilon>0$, we may take $n_{1}$ such as

$$
P_{r}\left(\Omega_{n_{1}}^{(1)}\right) \geqq c-\varepsilon / 2 \text {. }
$$

Let us put

$$
\Omega_{r_{i}}=\left\{\omega \in \Omega^{*} ; X\left(r_{i}, \omega\right)>\sqrt{r_{i}} \phi\left(r_{i}\right)\right\}
$$

Then we have

$$
\bigcup_{r_{i} \leqq n} \Omega_{r_{i}}=Q_{n_{1}}^{(1)}
$$

so that, if $i_{1}$ is sufficiently large, we obtain

$$
\operatorname{Pr}\left(\bigcup_{i \leqq i_{1}} \Omega_{r_{i}}\right) \gtrsim P_{r}\left(\Omega_{n_{1}}^{(1)}\right)-\varepsilon / 2 \gtrsim c-\varepsilon .
$$

Rearranging $\left\{r_{i} ; i \leqq i_{1}\right\}$ according to the order of magnitude, we obtain the set $\left\{t_{1}, \ldots, t_{i_{1}}\right\}$. Again we shall adopt the following definition:

$$
\Omega_{n}^{(2)}=\left\{\begin{array}{cl}
\left\{\omega \in \Omega^{*} ;{ }^{\exists} r_{i}, \max \left(i_{1}, t_{i_{1}}\right)<r_{i} \leqq n\right. & \text { and } \\
\left.X\left(r_{i}, \omega\right)>\sqrt{r_{i} \phi}\left(r_{i}\right)\right\} & \text { if } n>\max \left(i_{1}, t_{i_{1}}\right), \\
\text { empty set } & \text { otherwise. }
\end{array}\right.
$$

Then by (6)

$$
\bigcup_{n} \Omega_{n}^{(2)}=\Omega^{*}, \quad \Omega_{1}^{(2)} \cong \Omega_{2}^{(2)} \subseteq \ldots \subseteq \Omega_{n}^{(2)} \subseteq \ldots .
$$

Accordingly there exists $n_{2}$ such that

and

$$
P_{r}\left(\Omega_{n_{2}}^{(2)}\right) \gtrsim c-\varepsilon^{2} / 2
$$

$$
\bigcup_{\max \left(i_{1}, t_{\left.i_{1}\right)<r_{i} \equiv n_{2}}\right.} \Omega_{r_{i}}=\Omega_{n_{2}}^{(2)} .
$$


Therefore, if $i_{2}$ is sufficiently large, we have

$$
\operatorname{Pr}\left(\bigcup_{i_{1}<i \leqq i_{2}} \Omega_{r_{i}}\right) \geqslant P_{r}\left(\Omega_{n_{3}}^{(2)}\right)-\varepsilon^{2} / 2 \supseteq c-\varepsilon^{2} .
$$

By the same method as in the previous discussion we have a monotone sequence $\left\{t_{i_{1}+1}, \ldots, t_{i_{2}}\right\}$. Repeating this process, we have a monotone sequence such that

$$
\begin{aligned}
t_{1}<t_{2}<\ldots<t_{i_{1}}<\ldots<t_{i_{j}}<t_{i_{j}+1} \ldots & <t_{i_{j+1}}<\ldots, \\
& \left(t_{i} \rightarrow \infty \text { as } i \rightarrow \infty\right)
\end{aligned}
$$

and

$$
\operatorname{Pr}\left(\underset{i_{j-1}<i \leqq i j}{\bigcup} \Omega_{t_{i}}\right) \geqq c-\varepsilon^{j}
$$

Hence, if $\varepsilon<c / 2$, we obtain

$$
P_{r}\left(\bigcap_{j} \bigcup_{i j-1<i \leqq i j} \Omega_{t_{i}}\right) \geq c-\left(\varepsilon+\varepsilon^{2}+\ldots+\varepsilon^{n}+\ldots\right)=c-\frac{\varepsilon}{1-\varepsilon}>0 .
$$

(9) and (14) show that $\left\{\phi_{i}\right\}$ does not belong to the upper class with respect to $\left\{X_{i}\right\}$.

According to the following lemma which will be proved after the method of W. Feller, we can exchange in Lemma 1 the condition " $\left\{\phi_{k}\right\}$ does not belong to the upper class" by the condition " $\left\{\phi_{k}\right\}$ belongs to the lower class."

Lemma 2. Let the conditions in Theorem 1 be satisfied. Let $\left\{t_{k}\right\}$ be a monotone increasing sequence such that $t_{k} \rightarrow \infty \quad$ (as $\left.k \rightarrow \infty\right)$ and $t_{k}-t_{k-1} \leqq \alpha \leqq 1$. Then the monotone increasing sequence $\left\{\phi_{k}=\phi\left(t_{k}\right)\right\}$ belongs to the upper (lower) class with respect to $\left\{X_{k} ; X_{k}=X\left(t_{k}\right)-X\left(t_{k-1}\right)\right\}$ if, and only if,

$$
\sum_{k} \frac{t_{k}-t_{k-1}}{t_{k}} \phi_{k} e^{-\frac{1}{2} \phi_{k}^{2}} \in \mathbb{(}(\mathfrak{D}) .
$$

Theorem 1 is a simple corollary to Lemma 1 and Lemma 2.

\section{Proof of Theorem 1.}

a) The case of convergence. Let us suppose that $\phi(t)$ does not belong to the upper class. Then, according to Lemma 1 and Lemma 2, there exists a monotone increasing sequence $\left\{t_{k}\right\}$ such that $t_{k}-t_{k-1} \leqq \alpha$ and $\left\{\phi_{k}=\phi\left(t_{k}\right)\right\}$ belongs to the lower class with respect to $\left\{X_{k} ; X_{k}=X\left(t_{k}\right)-X\left(t_{k-1}\right)\right\}$. Hence by Lemma 2

$$
\sum_{k} \frac{t_{k}-t_{k-1}}{t_{k}} \phi_{k} e^{-\frac{1}{2} \phi_{k}^{2}} \in \mathscr{D}
$$

On the other hand, by the monotony of $\phi(t)$ and the assumption of convergence,

$$
\sum_{k} \frac{t_{k}-t_{k-1}}{t_{k}} \phi_{k} e^{-\frac{1}{2} \phi_{k}^{2}} \leqq \sum_{k} \int_{t_{k-1}}^{t_{k}} \frac{1}{t} \phi(t) e^{-\frac{1}{2} \phi^{2}(t)} d t
$$




$$
=\int^{\infty} \frac{1}{t} \phi(t) e^{-\frac{1}{2}-\phi^{2}(t)} d t \in(\zeta .
$$

This is a contradicition. So $\phi(t)$ must belong to the upper class.

b) The case of divergence. Let us consider the monotone increasing sequences $\left\{t_{k}=k \alpha\right\}$ and $\left\{\phi_{k}=\phi\left(t_{k}\right)\right\}$. Then we have

$$
\begin{aligned}
\int^{\infty} \frac{1}{\mathrm{t}} \phi(t) e^{-\frac{1}{2} \phi^{2}(t)} d t & =\sum_{k} \int_{t_{k-1}}^{t_{k}} \frac{1}{t} \phi(t) e^{-\frac{1}{2} \phi^{2}(t)} d t \\
& \leqq \sum_{k} \frac{t_{k}-t_{k-1}}{t_{k-1}} \phi_{k-1} e^{-\frac{1}{2} \phi_{k-1}^{2}} \\
& =\sum_{k} \frac{t_{k}-t_{k-1}}{t_{k}} \phi_{k} e^{-\frac{1}{2} \phi_{k}^{2}} .
\end{aligned}
$$

Thus the divergence of the integrals yields that of the series (15). Therefore, by Lemma $2,\left\{\phi_{k}\right\}$ must belong to the lower class and accordingly $\phi(t)$ belongs to the lower class with respect to the process $\{X(t, \omega)\}$.

Now our purpose is to prove Lemma 2. We put

$$
\eta_{k}^{2}=\left\{\begin{array}{lc}
t_{k}\left(\log \log t_{k}\right)^{-3} & \text { for } t_{k}>80, \\
\text { arbitrary in such a way that }\left\{\eta_{k}\right\} & \text { becomes a } \\
\text { monotone increasing sequence } & \text { for } t_{k} \leqq 80 .
\end{array}\right.
$$

Furthermore we put

$$
\begin{gathered}
P_{r}\left\{X_{k} \leqq x\right\}=F_{k}(x), \\
b_{k}=\int_{|x|<\eta_{k}} x^{2} d F_{k}(x), \\
B_{n}=\sum_{k=1}^{n} b_{k}, \\
\mu_{k}^{\prime}=-\int_{|x|<r_{k}} x d F(x), \quad \mu \mu_{k}^{\prime \prime}=-\int_{\eta_{h} \leqq|x|<t_{k}^{1 / 2}} x d F_{k}(x), \\
\mu_{k}^{\prime \prime \prime}=-\int_{t_{k}^{1 / 2} \leqq|x|} x d F_{k}(x)
\end{gathered}
$$

and

$$
\sigma_{k}^{2}=b_{k}-\mu_{k}^{\prime 2}, \quad s_{n}^{2}=\sum_{k=1}^{n} \sigma_{k}^{2} .
$$

We shall introduce three new sequences of random variables as follows;

$$
\begin{aligned}
& X_{k}^{\prime}= \begin{cases}X_{k}+\mu_{k}^{\prime} & \text { if }\left|X_{k}\right|<\eta_{k} \\
\mu_{k}^{\prime} & \text { otherwise, }\end{cases} \\
& X_{k}^{\prime \prime}= \begin{cases}X_{k}+\mu_{k}^{\prime \prime} & \text { if } \eta_{k} \leqq\left|X_{k}\right|<t_{k}^{1 / 2} \\
\mu_{k}^{\prime \prime} & \text { otherwise, }\end{cases}
\end{aligned}
$$




$$
X_{k}^{\prime \prime \prime}= \begin{cases}X_{k}+\mu_{k}^{\prime \prime \prime} & \text { if }\left|X_{k}\right| \geqslant t_{k}^{1 / 2} \\ \mu_{k}^{\prime \prime \prime} & \text { otherwise. }\end{cases}
$$

Then we have

$$
X_{k}=X_{k}^{\prime}+X_{k}^{\prime \prime}+X_{k}^{\prime \prime \prime},
$$

and the variables of each of the three sequences are mutually independent. Moreover

$$
E\left(X_{k}\right)=E\left(X_{k}^{\prime}\right)=E\left(X_{k}^{\prime \prime}\right)=E\left(X_{k}^{\prime \prime \prime}\right)=0
$$

and

$$
V\left(X_{k}^{\prime}\right)=\sigma_{k}^{2}
$$

If we define $S_{n}^{\prime}$ as follows

$$
S_{n}^{\prime}=X_{1}^{\prime}+X_{2}^{\prime}+\ldots+X_{n}^{\prime},
$$

(the sums $S_{n}^{\prime \prime}$ and $S_{n}^{\prime \prime \prime}$ are defined similarly), then we have

$$
V\left(S_{n}^{\prime}\right)=s_{n}^{2}
$$

LeMma 3. With probability one

$$
S_{n}^{\prime \prime \prime}=\mathrm{O}\left(t_{n}^{1 / 2}\left(\log \log t_{n}\right)^{-1 / 2}\right) .
$$

Proof. From the assumption (3) we have

$$
\begin{aligned}
\sum_{k} P_{r}\left\{X k_{k}^{\prime \prime} \neq \mu_{k}^{\prime \prime \prime}\right\} & =\sum_{k} \int_{|x| \geqq t_{k}^{1 / 2}} d F_{k}(x)=\mathrm{O}(1) \sum_{k}\left(t_{k}-t_{k-1}\right) \int_{|x| \Xi t_{k}^{1 / 2}} d V_{1}(x) \\
& =\mathrm{O}(1) \sum_{k=1}^{\infty} \sum_{j=k}^{\infty}\left(t_{k}-t_{k-1}\right) \int_{t_{j}^{1 / 2} \leqq|x|<t_{j+1}^{1 / 2}} d V_{1}(x) \\
& =\mathrm{O}(1) \sum_{j=1}^{\infty} \sum_{k=1}^{j}\left(t_{k}-t_{k-1}\right) \int_{t_{j}^{1 / 2} \leqq|x|<t_{j+1}^{1 / 2}} d V_{1}(x) \\
& =\mathrm{O}(1) \sum_{j=1}^{\infty} t_{j} \int_{t_{j}^{1 / 2} \leqq|x|<t_{j+1}^{1 / 2}} d V_{1}(x)=\mathrm{O}(1) \int_{-\infty}^{\infty} x^{2} d V_{1}(x)<\infty .
\end{aligned}
$$

Thus, by Borel-Cantelli's lemma, it follows that with probability one there will be only finitely many $k$ such that $X_{k}^{\prime \prime \prime} \neq \mu_{k}^{\prime \prime \prime}$. So, by the assumptions (1) and (2), we have

$$
\begin{aligned}
\left|S_{n}^{\prime \prime \prime}\right|= & \mathrm{O}(1)\left\{1+\left|\sum_{k=1}^{n} \int_{|x| \geqq t_{k}^{1 / 2}} x d F_{k}(x)\right|\right\}=\mathrm{O}(1)\left\{1+\sum_{k=1}^{n}\left(t_{k}-t_{k-1}\right) \int_{|x| \geqq t_{k}^{1 / 2}}|x| d V_{1}(x)\right\} \\
= & \mathrm{O}(1)\left\{1+\sum_{k=1}^{n} \sum_{j=k}^{\infty}\left(t_{k}-t_{k-1}\right) \int_{t_{j}^{1 / 2} \leqq|x|<t_{j+1}^{1 / 2}}|x| d V_{1}(x)\right\} \\
= & \mathrm{O}(1)\left\{1+\sum_{j=1}^{n-1} \sum_{k=1}^{j}\left(t_{k}-t_{k-1}\right) \int_{t_{j}^{1 / 2} \leqq|x|<t_{j+1}^{1 / 2}}|x| d V_{1}(x)\right. \\
& \left.+\sum_{j=n}^{\infty} \sum_{k=1}^{n}\left(t_{k}-t_{k-1}\right) \int_{t_{j}^{1 / 2} \leqq|x|<t_{j+1}^{1 / 2}}|x| d V_{1}(x)\right\}
\end{aligned}
$$




$$
\begin{aligned}
& =\mathrm{O}(1)\left\{1+\sum_{j=1}^{n-1} t_{j} \int_{t_{j}^{1 / 2} \leqq|x|<t_{j+1}^{1 / 2}}|x| d V_{1}(x)+\sum_{j=n}^{\infty} t_{n} \int_{t_{j}^{1 / 2} \leqq|x|<t_{+1}^{13 / 2}}|x| d V_{1}(x)\right\} \\
& =\mathrm{O}(1)\left\{1+\int_{|x|<t_{n}^{1 / 2}}|x|^{3} d V_{1}(x)+t_{n} \int_{t_{n}^{1 / 2} \leqq|x|}|x| d V_{1}(x)\right\} \\
& =\mathrm{O}(1)\left(\frac{t_{n}}{\log \log t_{n}}\right)^{1 / 2}
\end{aligned}
$$

This proves the lemma.

\section{LEMMA 4. With probability one}

$$
S_{n}^{\prime \prime}=\mathrm{O}\left(\left(t_{n} \log \log \log t_{n}\right)^{1 / 2}\right) .
$$

Proof. According to a theorem of L. Kronecker," it will be sufficient to prove that the series

$$
\sum_{n} \frac{1}{\left(t_{n} \log \log \log t_{n}\right)^{1 / 2}} X_{n}^{\prime \prime}
$$

converges with probability one. By a theorem of Khintchine and Kolmogoroff, it is sufficient to show that

$$
\sum_{n} \frac{1}{t_{n} \log \log \log t_{n}} E\left(X_{n}^{\prime \prime^{2}}\right) \in \Subset .
$$

To prove (33) we shall consider the following function

$$
S(n)=\min _{k \in T_{n}} k, \quad T_{n}=\left\{k ; \frac{t_{k}^{1 / 2}}{\left(\log \log t_{k}\right)^{3 / 2}}>t_{n}^{1 / 2}\right\} .
$$

Obviously $S(n)$ is monotone non-decreasing. Hence we can define the inverse function of $S(n)$ as follows

$$
S^{-1}(n)=\min _{S(l) \geqq n} l
$$

By the definition (23), we obtain

$$
\begin{aligned}
\sum_{n} \frac{1}{t_{n} \log _{(3)} t_{n}} E\left(X_{n}^{\prime \prime 2}\right) & \leqq \sum_{n} \frac{1}{t_{n} \log _{(3)} t_{n}} \int_{\eta_{n} \leqq|x|<t_{n}^{1 / 2}} x^{2} d F_{n}(x) \\
& =\mathrm{O}(1) \sum_{n} \frac{t_{n}-t_{n-1}}{t_{n} \log _{(3)} t_{n}} \int_{\eta_{n} \leqq|x|<t_{n}^{1 / 2}} x^{2} d V_{1}(x) \\
& =\mathrm{O}(1) \sum_{n} \frac{i_{n}-t_{n-1}}{t_{n} \log _{(3)} t_{n}} \sum_{k=n}^{S(n)} \int_{\eta_{k} \leqq|x|<\eta_{k+1}} x^{2} d V_{1}(x) \\
& =\mathrm{O}(1) \sum_{k} \int_{\eta_{k} \leqq|x|<\eta_{k+1}} x^{2} d V_{1}(x) \sum_{n=S^{-1}(k)}^{k} \frac{t_{n}-t_{n-1}}{t_{n} \log _{(3)} t_{n}}
\end{aligned}
$$

i) K. Knopp: Theorie und Anwendung der Unendlichen Reihen, 2 ed., Beriin, 1924, p. 127.

8) A. Kolmogoroff: Grundbegriffe der Wahrscheinlichkeitsrechung, Berlin. 1933, p. 59. 


$$
=\mathrm{O}(1) \sum_{k} \int_{\eta_{k} \leqq 1: x \mid<\tau_{i k+1}} x^{2} d V_{1}(x)<\infty,
$$

where $\log _{(k)}$ denotes the $k$-times iterated logarithm. This proves the lemma.

LEMMA 5. For any $\delta>0$ the probability is zero that there exist infinite many $n$ for which the inequalities

$$
S_{n}^{\prime \prime}>\delta t_{n}^{1 / 2} /\left(\log \log t_{n}\right)^{1 / 2}
$$

and

$$
S_{n}^{\prime}>\delta\left(t_{n} \log \log t_{n}\right)^{1 / 2}
$$

hold simultaneously.

Froof. Let us denote by $A_{n}$ the event that there exists at least one $t_{k}$ such that

$$
10 t_{n} \log _{(2)}^{-3} t_{n}<t_{k} \leqq t_{n} \text { and } X_{k}^{\prime \prime} \neq \mu_{k}^{\prime \prime},
$$

and by $\bar{A}_{n}$ its complementary event. Choosing $m$ for which $\left[t_{m}\right]=\left[10 t_{n} \log _{(2)}^{-3} t_{n}\right]^{91}$ holds, we have

$$
\begin{aligned}
\left|\sum_{k=m}^{n} \mu_{k}^{\prime \prime}\right| & =\mathrm{O}(1) \sum_{k=m}^{n}\left(t_{k}-t_{k-1}\right) \int_{r_{k} \leqq|x|-t_{k}^{1 / 2}}|x| d V_{1}(x) \\
& =\mathrm{O}(1) \sum_{k=m}^{n}\left(t_{k}-t_{k-1}\right) \frac{1}{\gamma_{k}\left(\log _{(2)} \eta_{k}\right)^{2+\varepsilon}} \\
& =\mathrm{O}(1) \sum_{k=m}^{n} \frac{t_{k}-t_{k-1}}{t_{k}^{1 / 2}\left(\log _{(2)} t_{k}\right)^{1 / 2+\varepsilon}} \\
& =\mathrm{O}(1) \frac{t_{1}^{i_{1} / 2}}{\left(\log \log t_{n}\right)^{1 / 2+\varepsilon}} .
\end{aligned}
$$

Accordingly, if $\bar{A}_{n}$ occurs, then we have by Lemma 4

$$
\begin{aligned}
S_{n}^{\prime \prime} & =S_{m}^{\prime \prime}+\left(S_{n}^{\prime \prime}-S_{m}^{\prime \prime}\right)=O\left(\left(t_{m} \log _{(3)} t_{m}\right)^{1 / 2}\right)+O\left(\begin{array}{c}
t_{n}^{1 / 2} \\
\left(\log \log t_{n}\right)^{1 / 2+\varepsilon}
\end{array}\right) \\
& =o\left(t_{n}^{1 / 2} /\left(\log \log t_{n}\right)^{1 / 2}\right) .
\end{aligned}
$$

This excludes (36). Therefore, for sufficiently large $n$, the event (36) will occur only in conjunction with the event $A_{n}$ with probability one. Let $B_{n}$ denote the event of a simultaneous realization of (37) and $A_{n}$. It suffices to prove that the probability that $B_{n}$ occurs for infinitely many $n$ is zero. To this purpose, we consider the event

$$
C_{\nu}=\sum_{e^{\nu-1}<\eta_{n} \leqq e^{\nu}} B_{n}
$$

which implies the realization of at least one $B_{n}$ with

9) $[x]$ denotes the largest integer which does not exceed $x$. 


$$
e^{i-1}<t_{n} \leqq e^{\nu} .
$$

Our lemma will be proved if we show that

$$
\sum_{\nu} \operatorname{Pr}_{r}\left(C_{2}\right)<\infty \text {. }
$$

Put

$$
P_{v}=\sum_{e^{\nu} \log ^{-3} v<t_{k} \cong \theta^{\nu}} P_{r}\left\{X_{k}^{\prime \prime} \neq \mu_{R}^{\prime \prime}\right\} .
$$

Then we obtain

$$
\sum_{\nu} \frac{P_{v}}{(\log \nu)^{100}}<\infty^{10)}
$$

and

$$
P_{r}\left(C_{\nu}\right)=\mathrm{O}(1) P_{\nu} /(\log \nu)^{10011)}
$$

Accordingly the series (41) converges.

LEMMA 6. For any monotone increasing sequence $\left\{\phi_{n}\right\}$ the divergence (convergence) of the series (15) is a necessary and sufficient condition that with probability one the inequality

$$
\sum_{k=1}^{n} X_{k}>B_{n}^{1 / 2} \phi_{n}
$$

be satisfied for infinitely (only finitely) many $n$.

Proof. Without loss of generality, we may assume that

$$
\log \log t_{n} \leqq \phi_{n}^{2} \leqq 4 \log \log t_{n} .^{12}
$$

If $a$ and $b$ are sufficiently large and $b-a \geqslant N$, then we have by the assumption (3)

$$
\int_{a}^{b} x^{2} d F_{n}(x)=\mathrm{O}(1)\left(t_{n}-t_{n-1}\right) \int_{a}^{b} x^{2} d V_{1}(x)
$$

So we have

$$
\begin{aligned}
\sigma_{n}^{2} & =b_{n}-\mu t_{n}^{\prime}=\int_{|x|<\eta_{n}} x^{2} d F_{n}(x)-\left(\int_{|x|<\eta_{n}} x d F_{n}(x)\right)^{2} \\
& =t_{n}-t_{n-1}-\int_{|x| \geqq \eta_{n}} x^{2} d F_{n}(x)-\left(\int_{|\tau|<\eta_{n}} x d F_{n}(x)\right)^{2} \\
& =t_{n}-t_{n-1}-O(1)\left(t_{n}-t_{n-1}\right)\left(\log \log t_{n}\right)^{-(2+\varepsilon)} .
\end{aligned}
$$

Thus $t_{n}-t_{n-1} / \sigma_{n}^{2} \rightarrow 1$ and therefore $t_{n} / s_{n}^{2} \rightarrow 1$ as $n \rightarrow \infty$. So the divergence (convergence) of (15) is equivalent to

10) 11) 12) loc. cit. 1). 


$$
\sum_{n} \frac{\sigma_{n}^{2}}{s_{n}^{2}} \phi_{n} e^{-\frac{1}{2} \phi_{n}^{2}} \in \mathscr{D}(\mathbb{L})
$$

According to a theorem of W. Feller, ${ }^{13)}$ (47) implies that with probability one there are infinitely (only finitely) many $n$ such that

$$
S_{n}^{\prime}>s_{n}\left(\phi_{n}+c / \phi_{n}\right),
$$

where $c$ is an arbitrary constant. From the definition (19) and (21), we have

$$
B_{n}-s_{n}^{2}=\sum_{k=1}^{n} \mu_{k}^{\prime^{2}}
$$

and

$$
\left|\mu_{k}^{\prime}\right|=\left|\int_{|x| \geqq \eta_{k}} x d F_{k}(x)\right| \leqq \frac{1}{\eta_{k}} \int_{|x| \geqq \eta_{k}} x^{2} d F_{k}(x) \leqq \frac{t_{k}-t_{k-1}}{\eta_{k}} .
$$

Hence $B_{n}-s_{n}^{2}=\mathrm{O}\left(\left(\log t_{n}\right)^{2}\right)$ and we may take $B_{n}^{1 / 2}$ for $s_{n}$ in (48), so we have

$$
S_{n}^{\prime}>B_{n}^{1 / 2}\left(\phi_{n}+c / \phi_{n}\right) \text {. }
$$

Hence, using Lemma 3 and Lemma 5 , the divergence of (15) yields that with probability one there exist infinitely many $n$ for which the ineqalities

$$
S_{n}^{\prime}>B_{n}^{1 / 2}\left(\phi_{n}+c / \phi_{n}\right)
$$

and

$$
\left|S_{n}^{\prime \prime}+S_{n}^{\prime \prime \prime}\right|<M\left(t_{n} / \log \log t_{n}\right)^{i / 2}
$$

where $c$ is an arbitrary constant and $M$ is a sufficiently large number, hold simultaneously. Let us put $c=2 M$ in (49). Then we see that with probability one there exist infinitely many $n$ such that

$$
\sum_{k=1}^{n} X_{k}=S_{n}^{\prime}+S_{n}^{\prime \prime}+S_{n}^{\prime \prime \prime}>B_{n}^{1 / 2} \phi_{n} .
$$

Conversely if (51) holds for infinitely many $n$ with probability one, it follows, by (30) and (31), that with probability one

$$
S_{n}^{\prime}>\frac{1}{2} B_{n}^{1 / 2} \phi_{n}
$$

for infinitely many $n$ appearing in (51). From Lemma 5, it follows that with probability one there exist infinitely many $n$ for which (50) and (51) hold simultaneously, so that we have

$$
S_{n}^{\prime}>B_{n}^{1 / 2}\left(\phi_{n}-2 M / \phi_{n}\right)>s_{n}\left(\phi_{n}-2 M / \phi_{n}\right) \text {. }
$$

This means that $\left\{\phi_{n}-2 M / \phi_{n}\right\}$ belongs to the lower class with respect to $\left\{X_{n}^{\prime}\right\rangle$.

13) W. Feller: "The general form of the so-called law of the iterated logarithm." Trans. Amer. Math. Soc. vol. 54 (1943), pp. 373-402. 
Then, by a theorem of W. Feller, ${ }^{14)}$ we have

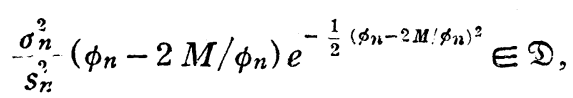

and accordingly

$$
\sum_{n} \frac{\sigma_{n}^{2}}{s_{n}^{2}} \phi_{n} e^{-\frac{1}{2} \phi_{n}^{2}} \in \mathbb{D}
$$

This is equivalent to the divergence of (15).

Now Lemma 2 will be proved easily.

Proof of Lemma 2. If the series (15) diverges, then it is clear, from Lemma 6, that for any constant $c$

$$
\sum_{k=1}^{n} X_{k}>B_{n}^{1 / 2}\left(\phi_{n}+c / \phi_{n}\right)
$$

will be satisfied for infinitely many $n$ with probability one. Therefore it is sufficient to show that

$$
t_{n}^{1 / 2}-B_{n}^{1 / 2}=\mathrm{O}\left(t_{n}^{1 / 2} / \phi_{n}^{2}\right)
$$

or, by $(46)$,

$$
t_{n}-B_{n}=\mathrm{O}\left(t_{n} / \log \log t_{n}\right)
$$

But we have

$$
\begin{aligned}
t_{n}-B_{n} & =\sum_{k=1}^{n} \int_{|x| \geqq \eta_{k}} x^{2} d F_{k}(x)=\mathrm{O}(1) \sum_{k=1}^{n}\left(t_{k}-t_{k-1}\right) \int_{|x| \geqq \eta_{k}} x^{2} d V_{1}(x) \\
& =\mathrm{O}(1)\left(t_{n} / \log \log t_{n}+t_{n} \int_{|x| \geqq t_{n}^{1 / 2} / \log _{(2)}^{2} t_{n}} x^{2} d V_{1}(x)\right)
\end{aligned}
$$

(the first term on the right is the contribution of the terms in the sum with $t_{k}<t_{n} / \log \log t_{n}$, and the integral is an upper bound for the contribution of the remaining terms). Hence, by the assumption (2), we have

$$
t_{n}-B_{n}=\mathrm{O}\left(t_{n} / \log \log t_{n}\right)
$$

The converse is trivial.

Proof of Theorem 2. In the proof of Theorem 1, the condition (1) was used to evaluate

$$
\sum_{k=1}^{n} \int_{|x| \geqq t_{k}^{1 / 2}} x d F_{k}(x)=\mathrm{O}\left(t_{n} / \log \log t_{n}\right)^{1 / 2} .
$$

But this is equal to zero in our case. Also the condition (2) was used to evaluate

11) loc. cit. 13). 


$$
\left|\sum_{k=1}^{n} \mu_{k}^{\prime \prime}\right|=\mathrm{O}\left(t_{n} /\left(\log \log t_{n}\right)^{1+2 \varepsilon}\right)^{1 / 2}
$$

and

$$
t_{n}-B_{n}=\mathrm{O}\left(t_{n} / \log \log t_{n}\right) .
$$

In our case the former is equal to zero and for the latter the condition (2)" is sufficient. These prove Theorem 2.

Proof of Theorem 3. Let $t=q / p$ be a rational number. Then we have

$$
\begin{aligned}
E\left((X(t))^{4}\right)= & \int_{-\infty}^{\infty} x^{4} d V_{t}(x)=\int(X(q / p))^{4} P(d \omega) \\
= & \int\{(X(1 / p)-X(0))+(X(2 / p)-X(1 / p)) \\
& +\ldots+(X(q / p)-X(q-1 / p))\}^{4} P(d \omega) \\
= & q \int_{-\infty}^{\infty} x^{4} d V_{1 / p}(x)+3 q(q-1) / p^{2} .
\end{aligned}
$$

Put $p=q$ and $E\left((X(1))^{4}\right)=a$. Then we obtain

$$
a=E\left((X(1))^{4}\right)=p \int_{-\infty}^{\infty} x^{4} d V_{1 / p}(x)+3(p-1) / p,
$$

and accordingly

$$
\int_{-\infty}^{\infty} x^{4} d V_{1 / p}(x)=(a-3(p-1) / p) / p
$$

Hence

$$
\begin{aligned}
E\left((X(t))^{4}\right) & =E\left((X(q / p))^{4}\right)=a q / p-3(1-q / p) q / p \\
& =a t-3 t(1-t) .
\end{aligned}
$$

Since $E\left((X(t))^{4}\right)$ is a monotone increasing function of $t$, (54) holds for any real number $t$.

Let us consider the sequence $\left\{X_{k}\right\}$ in Lemma 2. Using the notations in the previous proofs we have

$$
\begin{aligned}
P_{r}\left\{\left|X_{k}\right| \geqslant \eta_{k}\right\} & =\int_{|x| \geqq \eta_{k}} d F_{k}(x) \\
& \leqq \frac{1}{\eta_{k}^{4}} \int_{-\infty}^{\infty} x^{4} d F_{k}(x)<a\left(t_{k}-t_{k-1}\right) / \eta_{k}^{4}
\end{aligned}
$$

and so

$$
\sum_{k} P_{r}\left\{\left|X_{k}\right| \geqslant \eta_{k}\right\} \leqq a \sum_{k}\left(t_{k}-t_{k-1}\right)\left(\log \log t_{k}\right)^{6} / t_{k}^{2} \in \mathfrak{C} .
$$

According to Borel-Cantelli's lemrna, it follows that with probability one there will be only finitely many $k$ such that $\left|X_{k}\right| \geqslant \eta_{k}$. 
Put

$$
X_{k}^{\prime}= \begin{cases}X_{k}+\int_{|x| \geqq \eta_{i k}} x d F_{k}(x) & \text { if }\left|X_{k}\right|<\eta_{k} \\ \int_{|x| \geqq r_{k}} x d F_{k}(x) & \text { otherwise. }\end{cases}
$$

Then we have

$$
E\left(X_{k}^{\prime}\right)=0, \quad V\left(X_{k}^{\prime}\right)=t_{k}-t_{k-1}+\mathrm{O}(1)\left(t_{k}-t_{k-1}\right) / \eta_{k}^{2}
$$

and so

$$
t_{n}-\sum_{k=1}^{\prime \prime} V\left(X_{k}^{\prime}\right)=\mathrm{O}\left(\left(\log t_{n}\right)^{2}\right)
$$

On the other hand, we heve

$$
\sum_{k} \mid \int_{|x| \geqq \eta_{k}} x d F_{k}(x) \leqq a \sum_{k}\left(t_{k}-t_{k-1}\right) / \eta_{k}^{3} \in(.
$$

Hence

$$
\sum_{k=1}^{n} X_{k}=\sum_{k=1}^{n} X_{k}^{\prime}+O(1)
$$

By a theorem of W. Feller ${ }^{15)}$ the criterion (4) is valid for $\left\{X_{k}^{\prime}\right\}$ and so for $\left\{X_{k}\right\}$. Thus we may apply Lemma 2 , and Theorem 3 will be proved similarly as in the previous proof of Theorem 1 .

Mathematical Institute,

Nagoya University

15) loc. cit. 13). 